\title{
Price Transparency for COVID-19 Testing Among Top US Hospitals
}

J Gen Intern Med 36(1):245-7

DOI: $10.1007 / \mathrm{s} 11606-020-06197-\mathrm{Z}$

(c) Society of General Internal Medicine 2020

\section{INTRODUCTION}

On March 27, 2020, Congress passed the Coronavirus Aid, Relief, and Economic Security (CARES) Act in response to the COVID-19 pandemic and its economic repercussions. ${ }^{1}$ Under the CARES Act, private insurers are required to cover in vitro diagnostic testing for COVID-19 without patient cost sharing or other barriers during the public health emergency. To mitigate the risk of out-of-network billing for insurers that had not already negotiated rates, providers such as hospitals were required to publicly disclose the maximum "cash price" for tests online. Of note, this legislation does not prohibit providers from directly billing patients for testing. ${ }^{2}$

Price transparency may help promote widescale testing as policymakers seek to safely reopen the economy; in the coming months, an estimated 20 million tests per day will be necessary. ${ }^{3}$ However, there are reports that some providers may not be complying with price transparency mandates and instead imposing significant financial obligations on patients and payors. ${ }^{4}$

\section{METHODS}

We performed a cross-sectional analysis of online COVID-19 test price transparency among top US hospitals. We systematically reviewed the public websites of all hospitals in the 2019-2020 U.S. News and World Report "Best Regional Hospitals" list to determine whether the following information was available for in vitro COVID-19 tests: cash prices (yes/ no), hospital charges (yes/no), test type (molecular/serology/ unspecified; non-mutually exclusive). When available, cash prices represented the maximum amount payable to hospitals for testing by patients or insurers. For hospitals that did not list cash prices, hospital charges represented the maximum payable amount.

We collected information from dedicated hospital webpages on COVID-19 information, billing/price estimate webpages, public chargemasters, and web searches. For each

Received July 27, 2020

Accepted August 27, 2020

Published online September 18, 2020 hospital, we further extracted (as available) the lowest/highest cash prices and hospital charges for each test type. For hospitals that disclosed both cash prices and hospital charges, we estimated the corresponding out-of-network bill balance (defined as the difference between hospital charge minus the cash price) for each type of test offered.

We used descriptive statistics to summarize all information. Institutional Review Board approval was not required because this study analyzed publicly available data. All searches were completed between May 25, 2020, and May 29, 2020. We analyzed all data using Microsoft Excel Version 16.37 (Microsoft Corporation, Redmond, WA) and R version 3.6.0 (R Foundation for Statistical Computing, Vienna, Austria).

\section{RESULTS}

Approximately one-third $(n=181 / 566 ; 32.0 \%)$ of hospitals disclosed cash prices for in vitro COVID-19 testing (Fig. 1); $181(32.0 \%)$ listed only hospital charges and 204 (36.0\%) did not disclose pricing information. Among hospitals disclosing cash prices, the median lowest prices for molecular $(N=76)$ and serology $(N=36)$ were $\$ 67.90$ (interquartile range [IQR]: \$51.31-\$122.75) and \$57.00 (IQR: \$40.75-\$70.00), respectively. The median highest cash price for molecular tests was $\$ 100.00$ (IQR: $\$ 81.60-\$ 157.50$ ) with a maximum cash price of $\$ 525.00$.

Among hospitals disclosing only hospital charges, the median lowest charges for molecular $(N=181)$ and serology $(N=83)$ were $\$ 130.00$ (IQR: $\$ 100.00-\$ 195.62)$ and $\$ 100.00$ (IQR: \$65.00-\$153.00), respectively. The median highest hospital charge for molecular tests was $\$ 153.00$ (IQR: \$101.31-\$250.00) with a maximum hospital charge of $\$ 850.00$.

Among hospitals disclosing both cash prices and hospital charges, the median lowest bill balances for molecular $(N=$ $27)$ and serology $(N=14)$ were $\$ 66.96$ (IQR: $\$ 7.00-\$ 120.00)$ and \$6.00 (IQR: \$6.00-\$30.00), respectively. The median highest bill balance for molecular tests was $\$ 108.25$ (IQR: $\$ 10.00-\$ 234.00$ ) with a maximum bill balance of $\$ 383.96$.

\section{DISCUSSION}

Our analysis supports concerns about hospital compliance with federal regulations to disclose pricing information for COVID-19 testing. These findings may be partially explained 


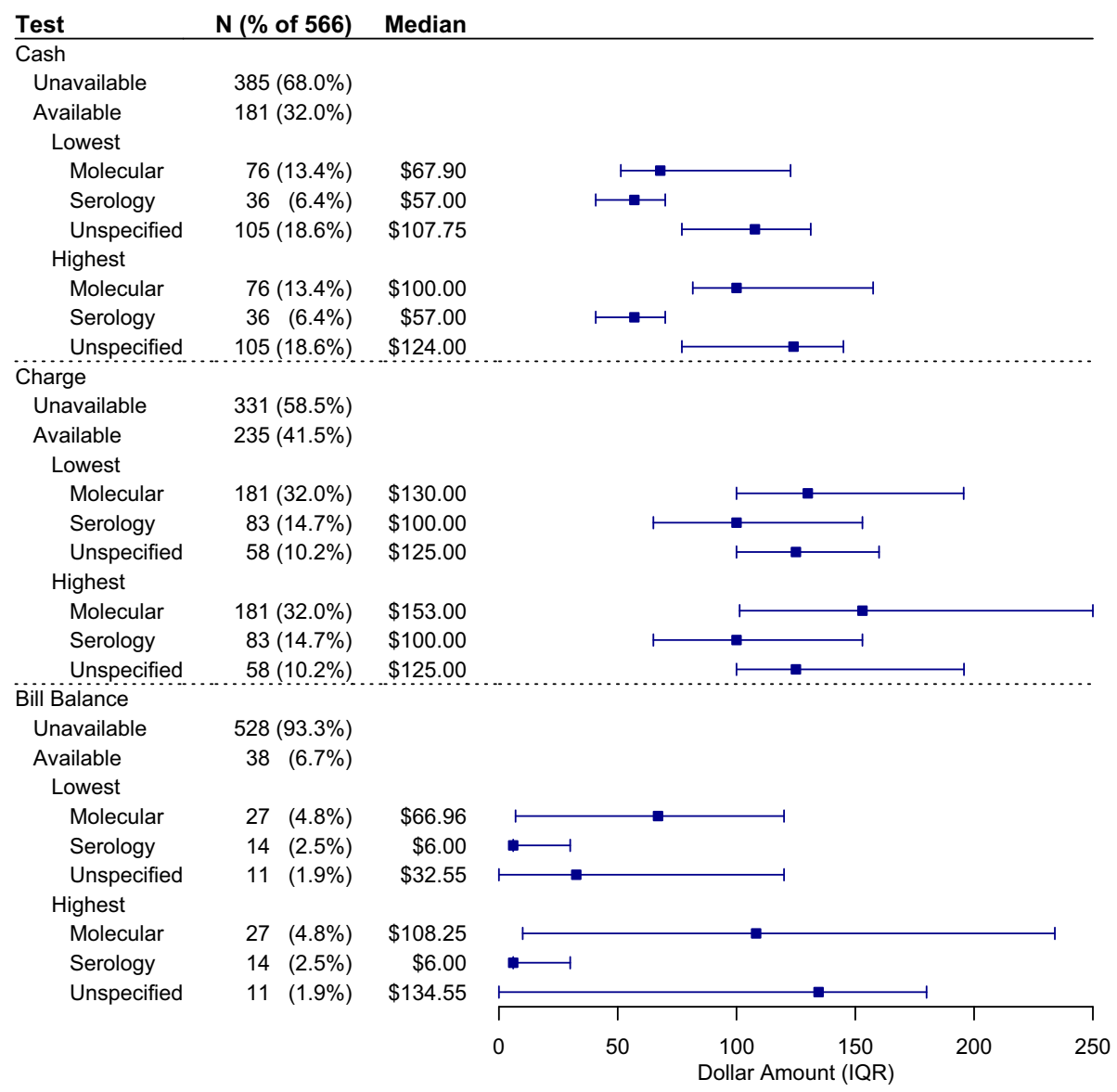

Figure 1 Price transparency for in vitro diagnostic COVID-19 testing among 2019-2020 U.S. World and News Report Best Regional Hospitals $(n=566)$. Caption: Excludes two hospitals that solely offered antigen testing for COVID-19.

by the relatively modest penalties ( $\$ 300$ per day) for noncompliance. ${ }^{1}$ To help patients and insurers better understand the price of testing, lawmakers could consider measures such as prohibiting providers from balance billing patients for testing or establishing price ceilings ${ }^{5}$ for private insurers using Medicare prices as a reference.

Our study has limitations. We restricted analysis to top US hospitals; thus, our findings are not generalizable to labs or other types of providers (e.g., urgent care centers). Furthermore, we may underestimate the overall level of price transparency for COVID-19 testing because information may be available through other means, such as phone or point-ofservice inquiries.

Lessening the financial uncertainties and burdens of COVID-19-related care will be critical as many Americans face the hardships of recession. Adoption of equitable payment policies for testing would be an important step towards protecting patients and public health during the ongoing crisis.

Data Access and Responsibility: Dr. Xiao had full access to all the data in the study and takes responsibility for the integrity of the data and the accuracy of the data analysis.

Roy Xiao, $M D, M S^{1,2}$

Vinay K. Rathi, $M D, M B A^{1,2}$
${ }^{1}$ Department of Otolaryngology - Head and Neck Surgery, Massachusetts Eye and Ear,

Boston, MA, USA

${ }^{2}$ Department of Otolaryngology - Head and Neck Surgery, Harvard Medical School,

Boston, MA, USA

Corresponding Author: Roy Xiao, MD, MS; Department of Otolaryngology - Head and Neck Surgery, Massachusetts Eye and Ear, Boston, MA, USA (e-mail: roy_xiao@meei.harvard.edu).

Author Contributions Drs. Xiao and Rathi were responsible for the conception and design of this work and manuscript drafting. Dr. Xiao was responsible for the acquisition of data and statistical analysis. All authors participated in the analysis and interpretation of the data and critically revised the manuscript for important intellectual content. Dr. Xiao is the guarantor.

\section{Compliance with Ethical Standards:}

Conflict of Interest: The authors declare that they do not have a conflict of interest.

\section{REFERENCES}

1. McConnell M. S.3548 - CARES Act. 2020.

2. Farmer B. Congress Said COVID-19 Tests Should Be Free - But Who's Paying? Kaiser Health New. 2020. 
3. Allen D, Block S, Cohen $\mathbf{J}$, et al. Roadmap to Pandemic Resilience Massive Scale Testing, Tracing, and Supported Isolation (TTSI) as the Path to Pandemic Resilience for a Free Society. Boston; 2020.

4. Kliff S. Most Coronavirus Tests Cost About $\$ 100$. Why Did One Cost \$2,315? The New York Times. 2020.
5. Adler L. How the CARES Act affects COVID-19 test pricing. Brookings. 2020.

Publisher's Note Springer Nature remains neutral with regard to jurisdictional claims in published maps and institutional affiliations. 